\title{
Correlation of gastrointestinal perforation location and amount of free air and ascites on CT imaging
}

\author{
Dionysios Drakopoulos ${ }^{1}$. Jacqueline Arcon ${ }^{1} \cdot$ Peter Freitag $^{1} \cdot$ Mostafa El-Ashmawy $^{1}$. Steven Lourens ${ }^{1}$.

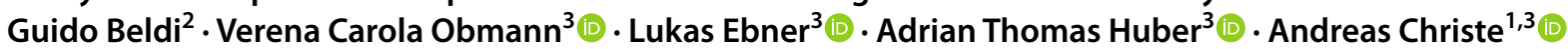

Received: 5 March 2021 / Revised: 5 May 2021 / Accepted: 19 May 2021 / Published online: 10 June 2021

(C) The Author(s) 2021

\begin{abstract}
Purpose To analyze the amount of free abdominal gas and ascites on computed tomography (CT) images relative to the location of a perforation.

Methods We retrospectively included 172 consecutive patients (93:79=m:f) with GIT perforation, who underwent abdominal surgery (ground truth for perforation location). The volume of free air and ascites were quantified on CT images by 4 radiologists and a semiautomated software. The relation of the perforation location (upper/lower GIT) and amount of free air and ascites was analyzed by the Mann-Whitney test. Furthermore, best volume cutoff for upper and lower GIT perforation, areas under the curve (AUC), and interreader volume agreement were assessed.

Results There was significantly more abdominal ascites with upper GIT perforation ( $333 \mathrm{ml}$, range 5 to $2000 \mathrm{ml}$ ) than with lower GIT perforation ( $100 \mathrm{ml}$, range 5 to $2000 \mathrm{ml}, p=0.022)$. The highest volume of free air was found with perforations of the stomach, descending colon and sigmoid colon. Significantly less free air was found with perforations of the small bowel and ascending colon compared to the aforementioned. An ascites volume $>333 \mathrm{ml}$ was associated with an upper GIT perforation demonstrating an AUC of $0.63 \pm 0.04$.

Conclusion Using a two-step process based on the volumes of free air and free fluid can help localizing the site of perforation to the upper, middle or lower GI tract.
\end{abstract}

\section{Graphic abstract}

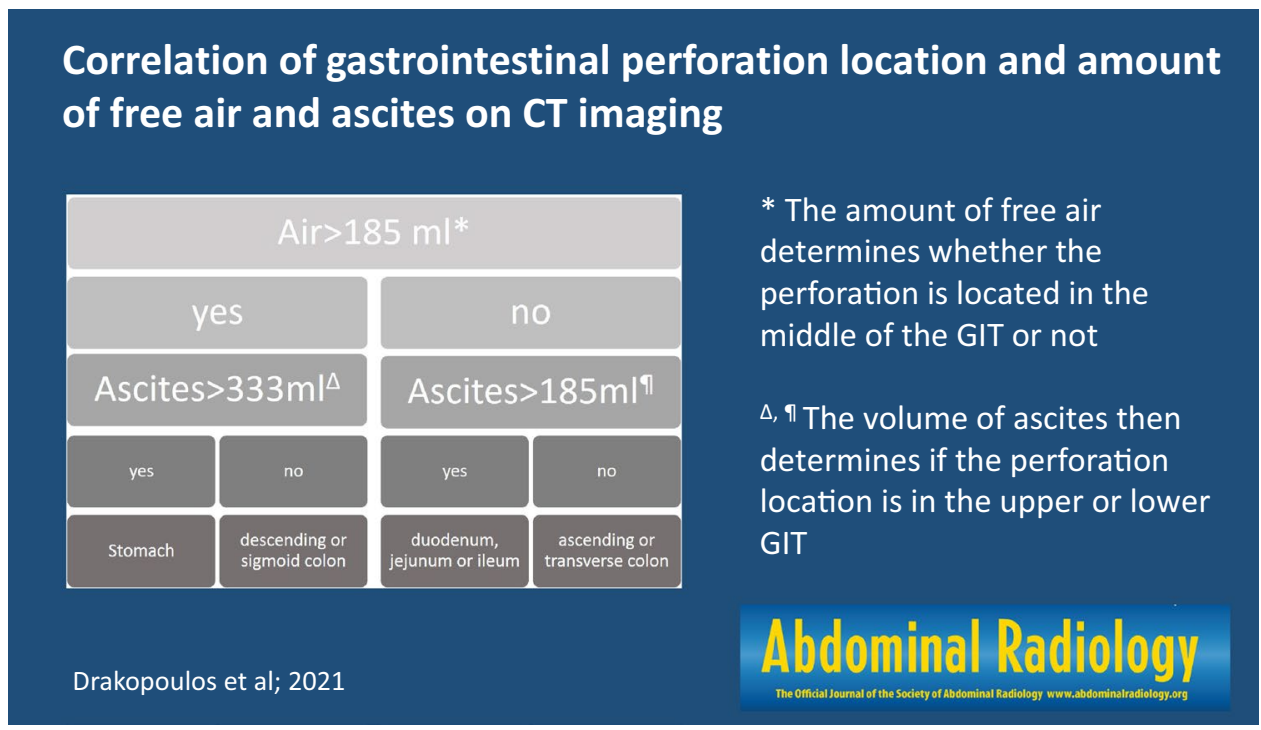

Keywords Gastrointestinal perforation $\cdot$ Abdominal CT imaging $\cdot$ CT volumetry $\cdot$ Volumetry of ascites $\cdot$ Volumetry of air

Extended author information available on the last page of the article 


\section{Introduction}

Breaching of the gastrointestinal (GI) tract wall can be due to ulcer disease, inflammatory disease, blunt or penetrating trauma, iatrogenic factors, a foreign body or a neoplasm [1-6]. The most important questions to be answered regard the identification of the presence, location, and cause of the perforation in order to perform the appropriate therapeutic procedure because gastrointestinal tract (GIT) perforation is a major life-threatening condition with high morbidity and mortality that requires emergency surgery; despite improvements in surgical and medical treatments, the overall mortality rate is $30 \%$, and the mortality rate of cases that also involve diffuse peritonitis is up to $70 \%$ [7-10]. Clinical diagnosis of the site of GIT perforation is difficult, as the symptoms may be nonspecific. The clinical presentation varies; esophageal perforations can present with acute chest pain, odynophagia and vomiting, gastroduodenal perforations cause acute, severe abdominal pain, while colonic perforations tend to follow a slower course of progression with secondary bacterial peritonitis or localized abscesses.

The presence of free intraperitoneal gas on a routine radiograph usually indicates bowel perforation. Some studies have shown that as little as $1 \mathrm{ml}$ of gas can be detected below the right hemidiaphragm on properly exposed erect chest radiographs [11]. Plain film radiography (erect chest and abdominal radiographs) is sensitive in only $50-70 \%$ of cases, and the site of perforation is almost never elucidated $[12,13]$. A left lateral decubitus film can also be used for the detection of small amounts of free air that may be interposed between the free edge of the liver and the lateral wall of the peritoneal cavity. When interpreting a right lateral decubitus image, gas within the stomach or colon may obscure small amounts of free air. Other modalities include ultrasound, which may be particularly useful in patient groups where the radiation burden should be limited, notably children and pregnant women. However, ultrasound should not be considered a first choice in excluding pneumoperitoneum [14]. Computed tomography (CT) is useful in detecting minute amounts of extraluminal gas $[15,16]$, the sensitivity of CT for free gas lies between 92 and 100\% [17-20] A study of multidetector CT showed $86 \%$ accuracy in identifying the site of perforation [21].

Time is of the essence in these patients. Knowing the exact location of GIT perforation is crucial for surgeons, since the operation time, as well as morbidity and mortality, can thereby be decreased.

In this study, we aimed to predict the location of perforations by analyzing the amount of free abdominal gas and ascites on CT images. Our hypothesis was that more free gas than ascites in the abdomen indicates an upper GIT perforation; and more ascites than gas indicates a lower GIT perforation due to peritonitis.

\section{Materials and methods}

The Cantonal Ethics Committee approved this retrospective study (Ethics Approval Nr. 2020-01279).

\section{Recruitment}

A full-text search for "perforation" in the radiological information system (RIS, GE Healthcare, Chicago, Illinois, USA) was performed between 01.01 .2003 and 01.01 .2020 by a $\mathrm{PhD}$ student. Patients who had been examined by abdominal $\mathrm{CT}$ in our emergency room with or without the use of contrast media and whose records included the word "perforation" in the radiological report were included.

Exclusion criteria:

(1) Patients without available operation report were excluded (not operated on, not operated in our hospital).

(2) Patients with no perforation in the radiological report were excluded ("suspicion of perforation" or "no signs of perforations", both showed in the radiological fulltext search).

(3) Perforation with obvious perforation locations like covert perforations or extraperitoneal perforations were excluded.

(4) Patients with other reasons for free air than GIT perforation were excluded like postinterventional (drainage) or postsurgical free gas; but also posttraumatic patients with perforating injuries (abdominal wall laceration/ defect).

(5) Patients with other reasons for ascites than GIT perforation were excluded, like liver cirrhosis, peritoneal carcinomatosis, pancreatitis and trauma with hemorrhagic ascites (trauma patients with hyperdense ascites were excluded, nontrauma patients with hyperdense ascites (blood or contrast) were included).

A total of 223 patients were found and matched to the surgical operation report from the CGM CLINICAL clinical information system (CompuGroup Medical Schweiz AG, Bern, Switzerland, Version: 7.16.1-5). The location of the perforation was extracted from the operation reports and represented the ground truth. After applying the exclusion criteria 172 patients remained for our study (Flowchart Fig. 1). The matching $\mathrm{CT}$ images were selected and anonymized by the PhD student and transferred to a read-out folder in our picture archiving and communication system (PACS, IDS7, 
Fig. 1 Flowchart for inclusion and exclusion of patients, preferred reporting items for systematic analyses (PRISMA). Covered perforations were excluded: the perforation is sealed off by adjacent organs, but the perforation location is obviously revealed by small air bubbles in close neighborhood of the perforation

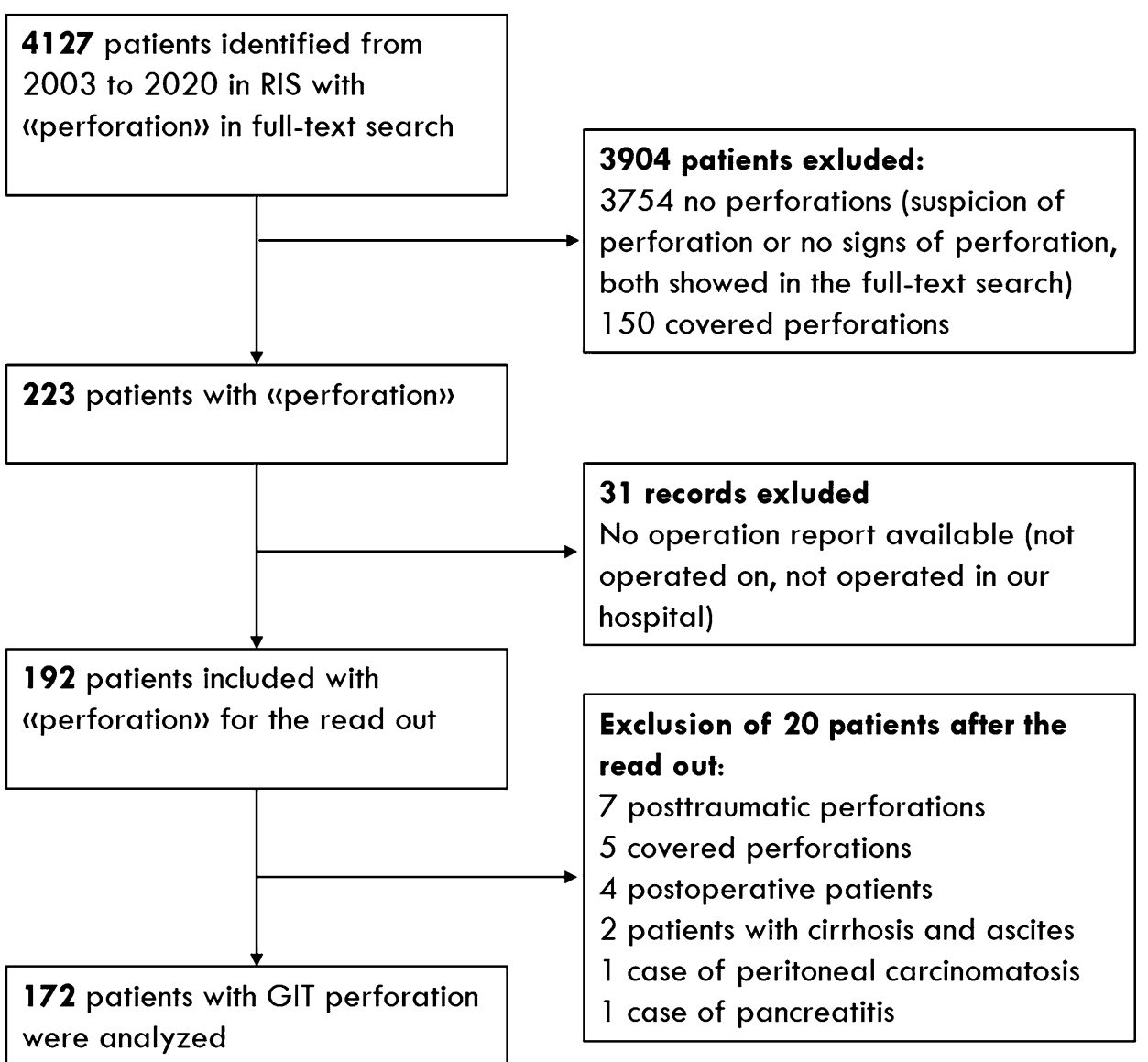

Sectra, Linköping, Sweden). Consecutive case numbering was assigned to each patient.

\section{Image acquisition}

Two different CT scanners (Siemens SOMATOM Sensation 16 and SOMATOM Definition Edge, both from Siemens Healthcare, Erlangen, Germany) were used. Before 2012, the older model SOMATOM sensation 16 applied 120-140 $\mathrm{kVp}, 160$ reference mAs tube current, $16 \times 0.75-\mathrm{mm}$ collimation, 1.15 pitch and standard filtered back projection with a slice thickness of 5 and $2 \mathrm{~mm}$. A total of $120 \mathrm{ml}$ of standard iodinated contrast medium (CM) with $300 \mathrm{mg} / \mathrm{ml}$ iodine (iobitridol, Xenetix 300; Guerbet, Aulnay-sous-Bois, France) was administered intravenously (i.v.) with an image acquisition delay of $60 \mathrm{~s}$ and a flow rate of $3 \mathrm{ml} / \mathrm{s}$. Telebrix gastro with an iodine concentration of $300 \mathrm{mg} / \mathrm{ml}$ (Megluminioxitalamat, Guerbet, Aulnay-sous-Bois, France) was used as oral and rectal CM: $24 \mathrm{ml} \mathrm{CM}$ was dissolved with $800 \mathrm{ml}$ tap water. This CM was orally administered $1 \mathrm{~h}$ prior to the CT exam and instilled rectally directly before the scan.

Since 2012, the new SOMATOM Definition Edge has used $100-140$ care $\mathrm{kVp}, 120-160$ reference $\mathrm{mAs}$ as the tube current, $128 \mathrm{~mm} \times 0.6 \mathrm{~mm}$ collimation, 0.6 pitch and iterative reconstruction (SAFIRE, level 3). Transverse images were reconstructed at intervals of 5 and $1 \mathrm{~mm}$. One hundred milliliters of Iomeron $400 \mathrm{mg} / \mathrm{ml}$ was injected intravenously with a flow rate of $3 \mathrm{~mL} / \mathrm{s}$. Data acquisition was started after $70 \mathrm{~s}$. No oral or rectal CM was used anymore. Before 2012 the gold standard in emergency abdominal CT imaging was IV, oral and rectal CM application [22, 23]. To save time for the critically ill patients our department changed the emergency CT protocols in 2012 to IV CM without oral or rectal CM application [24-26].

\section{Image analysis}

Images were reviewed by 4 board-certified radiologists (designated as 1,2,3, and 4) with 26, 33, 9, and 22 respective years of experience in abdominal imaging. Radiologists 1 and 2 analyzed 100 cases separately and radiologists 3 and 4 read 92 cases separately (different from the first 100 cases). All four readers were blinded from each other and to the perforation location. During the read out, a total of 20 patients had to be excluded (e.g., posttraumatic, postoperative and covert perforations, details in Flowchart of Fig. 1) leading to the remaining 172 study patients. The average volume of the paired readers was calculated for free air and ascites: 
The amount of free air and ascites was rated by visual comparison to the volume of cooking or drinking units: teaspoon $(5 \mathrm{ml})$, tablespoon $(15 \mathrm{ml})$, shot glass $(40 \mathrm{ml})$, small drinking glass $(1 \mathrm{dl}=100 \mathrm{ml})$, soda can $(333 \mathrm{ml})$, and PET bottle $(500 \mathrm{ml} / 1000 \mathrm{ml} / 1500 \mathrm{ml} / 2000 \mathrm{ml}$; PET: PolyEthylene Terephthalate plastic bottle). Two additional medical technicians from our imaging laboratory performed a semiautomated computer-aided volume measurement of free air and ascites from 35 of the first 100 cases and 30 of the following 92 patients using syngo.via (Siemens Healthcare $\mathrm{GmbH}$, Erlangen, Germany). Semiautomated means that the software uses a region-growing process that suggests the area of free air/ascites on the CT scan. The 3D annotation requires a time-consuming manual adjustment by the medical technician to differentiate air from feces and fat (or ascites from soft-tissue structures) (Fig. 2). On average the $3 \mathrm{D}$ analysis of one patient took $30 \mathrm{~min}$. Because of that time constraint we planned the 3D analysis only in one third of the patients, randomly. The semiautomated measurement was used for detecting accuracy and agreement of radiologists in estimating the volume of ascites and free air.

The human raters determined if there was more gas than ascites to test our hypothesis for an easy applicable tool without performing measurements by the radiologists. Furthermore, they estimated the most likely location of the perforation by using radiological criteria (most extensive free gas or air bubble accumulation, bowel wall thickening or discontinuation and most extensive mesenteric fat imbibition). They classified the perforation location as follows: (1) stomach, (2) duodenum, (3) jejunum, (4) ileum, (5) ascending colon, coecum or appendix, (6) transverse colon, (7) descending colon and (8) sigmoid colon or proximal rectum. In addition, the radiologists had to indicate their confidence level in rating the perforation location: $1=$ no confidence in localizing the perforation site; $2=$ some confidence; $3=50 \%: 50 \%$ confidence, $4=$ reasonably sure, $5=100 \%$ sure of the location.

For each reader, a master read-out Excel file was compiled and saved daily with a traceable calendar date on a server drive with restricted access for radiologists only. For each reader, a personal encoded read-out Excel file was compiled, containing only the patient code and the read-out variables (volume and location). The Excel sheets were stored in SharePoint of our hospital domain.

\section{Statistics}

The label "air scenario" was attributed to cases with more free air than ascites, and the "ascites scenario" was attributed to patients with more ascites than free air. Perforation locations in the (1) stomach, (2) duodenum, (3) jejunum, (4) ileum, (5) ascending colon, coecum or appendix, (6) transverse colon, (7) descending colon and (8) sigmoid colon or proximal rectum were pooled into upper GIT (1-4) and lower GIT (5-8) perforations. The prevalence of the perforation location was analyzed per GIT segment (1-8) by chi square testing. The median absolute volumes of air and ascites for perforations of each GIT segment were calculated by using the average volume estimates of both radiologists. Comparisons among the different segments were performed by using the rank sum test (Mann-Whitney independent testing). The volume of free air and ascites alone and the sum, the delta and the ratio of air and ascites were tested to classify the perforation location as upper or lower GIT using the rank sum test (Mann-Whitney independent testing). The "air scenario" and "ascites scenario" were analyzed by chi square testing as a sign of upper or lower GIT perforation. Receiver operating characteristic (ROC) statistics for the best volume cutoff for upper and lower GIT perforation were calculated, delivering individual sensitivities, specificities and areas under the curve (AUCs). Furthermore, interreader agreement was assessed for volume rating among the four radiologists, and the correlation coefficient and limits of agreement between the semiautomated and radiologist measurements were calculated. Due to the volume approach by drinking units used by the radiologists, the number of entries was limited, and the weighted kappa
Fig. 2 Semiautomated volumetry on axial slices of a perforation in the descending colon. A Preannotation and $\mathbf{B}$ postannotation of free air (asterisk, pink) with a total volume of $1972 \mathrm{ml}$ and $93 \mathrm{ml}$ of ascites (arrow, green)

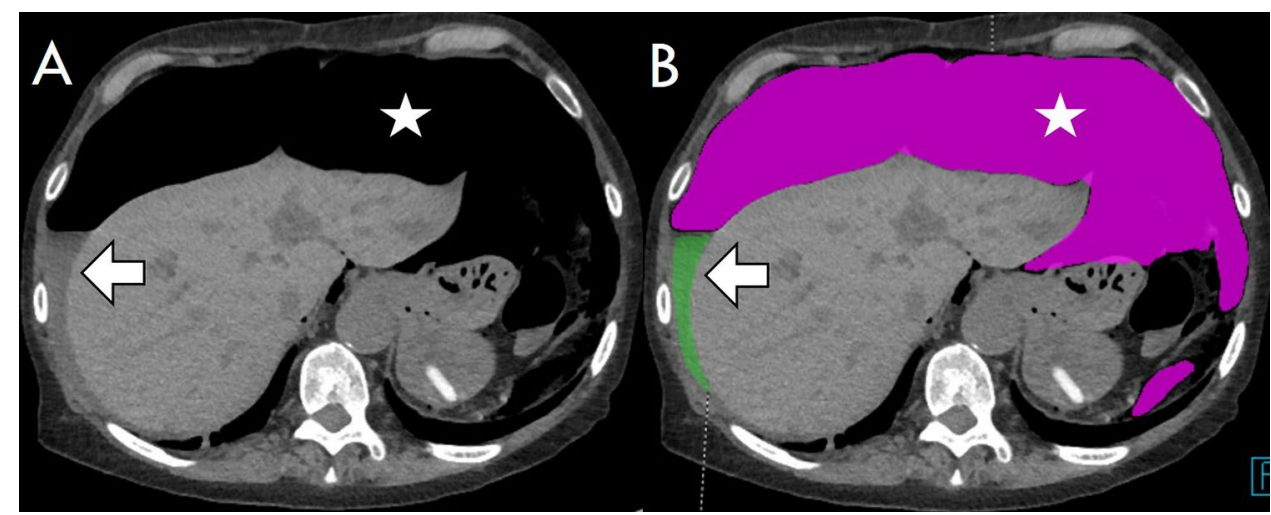


could be calculated. For the volume comparison with the machine (continuous volume spectrum), the correlation coefficient and the Bland-Altman agreement approach were used. For the following kappa classification of interrater agreement, $\kappa<0=$ poor agreement, $0.0-0.20=$ slight, $0.21-0.40=$ fair, $0.41-0.60=$ moderate, $0.61-0.80=$ substantial, and $0.81-1.00=($ almost $)$ perfect agreement was used. MedCalc (Version 7.6.0.0, Ostend, Belgium) was used for the statistical computation. A significance level of $p<0.05$ was applied. The radiologists' correct perforation location was expressed as the sensitivity for segmental classification and for upper/lower GIT classification. Furthermore, the radiologists' experience was compared for correct perforation location prediction (chi square testing).

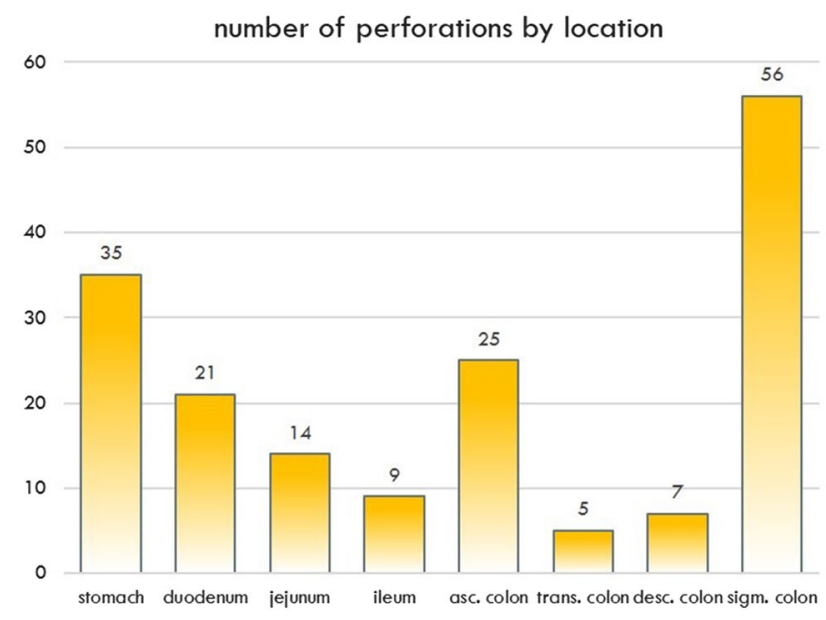

Fig. 3 Perforation by location

\section{Results}

Between 01.01.2003 and 01.01.2020, a total of 172 abdominal cases with GIT perforations were finally analyzed. The median age was 66.1 years (range 1.2 to 94.4 years), and the sex ratio was $93: 79=$ m:f. In $5 \%$ of our entire study population, CT scans were performed without IV contrast media. Thirty percent, $45 \%$ and $25 \%$ of the study patients received oral, both oral and rectal and no GIT contrast media, respectively.

\section{Location of the GIT perforation (consecutive study)}

A total of $54.1 \%(n=93)$ of all perforations were found in the upper GIT, and $45.9 \%(n=79)$ were found in the lower GIT. The top three locations were the sigmoid colon, stomach and ascending colon, with prevalences of $32.6 \%(n=56)$, $20.3 \%(n=35)$ and $14.5 \%(n=25)$, respectively. The transverse colon demonstrated significantly fewer perforations than the sigmoid colon $(2.9 \%, p<0.0001$, entire prevalence statistics shown in Fig. 3 and Table 1).

\section{Dependency of the perforation location and the amount of free air}

All of the patients demonstrated free air since this was an inclusion criterion. The median volume of free air was $174 \mathrm{ml}$ (percentiles $25-75 \%=40-417 \mathrm{ml}$ ) in perforations of the upper GIT, compared to $100 \mathrm{ml}$ (percentiles $25-75 \%=28-500 \mathrm{ml}$ ) in perforations of the lower GIT $(p=0.47)$. The highest volume of free air was found in perforations of the stomach, descending colon and sigmoid colon (333, 333, 143 ml, entire statistics in Table 1, Fig. 4 and 5). Significantly less free air was present in perforations
Table 1 Distribution of perforations with air and ascites volume (ml)

\begin{tabular}{|c|c|c|c|c|c|c|c|c|}
\hline \multicolumn{2}{|l|}{ PERFORATION } & \multirow{3}{*}{$N(\%)$} & \multicolumn{3}{|c|}{ AIR (ml) } & \multicolumn{3}{|c|}{ ASCITES (ml) } \\
\hline & \multirow[t]{2}{*}{$N$} & & \multirow[t]{2}{*}{ Median } & \multicolumn{2}{|c|}{ Percentiles } & \multirow[t]{2}{*}{ Median } & \multicolumn{2}{|c|}{ Percentiles } \\
\hline & & & & $25 \%$ & $75 \%$ & & $25 \%$ & $75 \%$ \\
\hline STOMACH & 35 & 20.3 & 333 & 119 & 500 & 417 & 100 & 750 \\
\hline DUODENUM & 21 & 12.2 & 70 & 40 & 225 & 333 & 58 & 750 \\
\hline JEJUNUM & 14 & 8.1 & 61 & 23 & 100 & 85 & 23 & 1500 \\
\hline ILEUM & 9 & 5.2 & 40 & 5 & 93 & 250 & 68 & 625 \\
\hline ASC. COL & 25 & 14.5 & 40 & 16 & 217 & 70 & 33 & 249 \\
\hline TRANS. COL & 5 & 2.9 & 100 & 54 & 750 & 100 & 55 & 1063 \\
\hline DESC. COL & 7 & 4.1 & 333 & 21 & 604 & 70 & 57 & 100 \\
\hline SIGMA/RECT & 56 & 32.6 & 143 & 40 & 500 & 180 & 28 & 333 \\
\hline upper GIT & 79 & 45.9 & 174 & 40 & 417 & 333 & 70 & 750 \\
\hline lower GIT & 93 & 54.1 & 100 & 28 & 500 & 100 & 31 & 333 \\
\hline
\end{tabular}

$N$ number of patients 


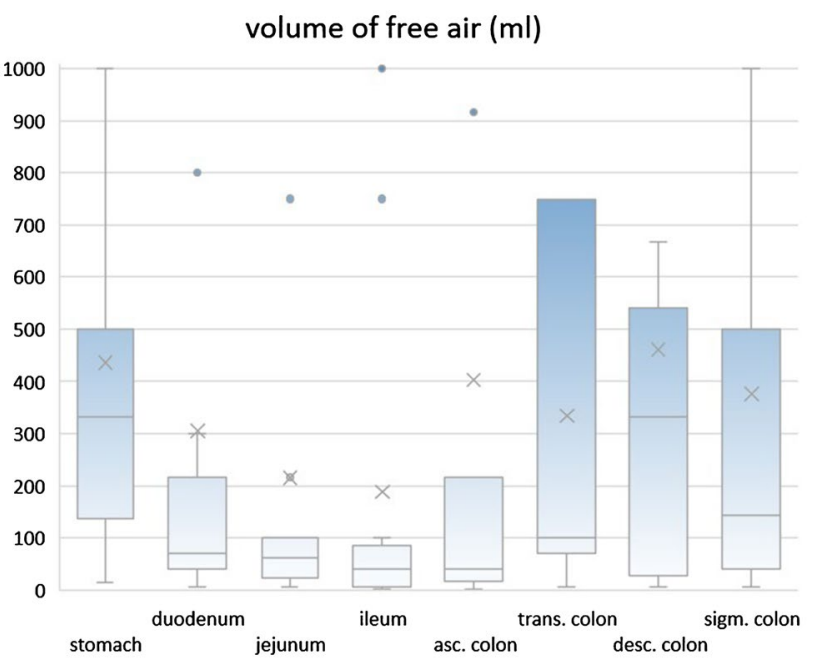

Fig. 4 Box-and-Whisker plot of the amount of free air per perforation location

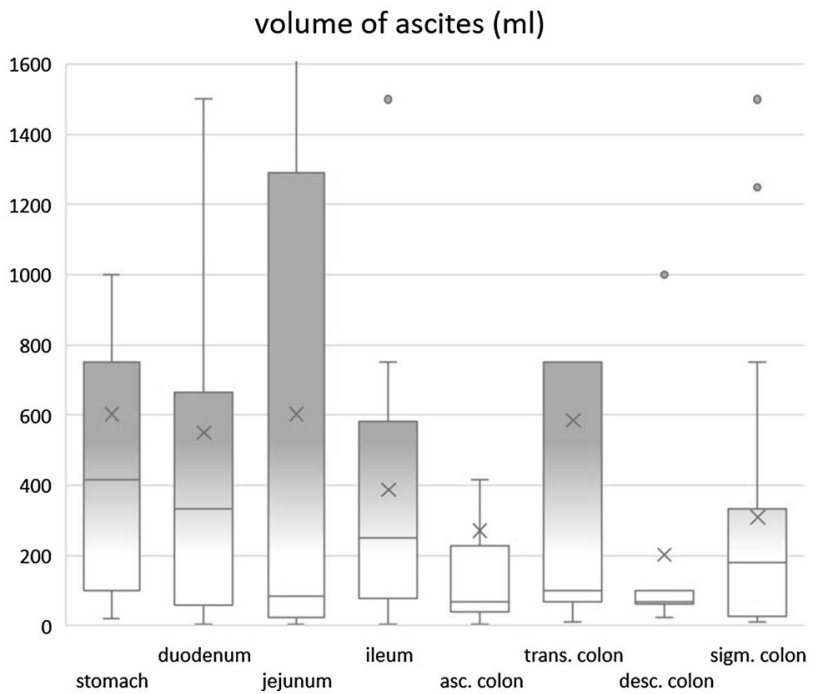

Fig. 5 Box-and-Whisker plot of the amount of ascites per perforation location

of the middle GI tract: duodenum, jejunum, ileum ascending and transverse colon leaked only 70, 61, 40, 40 and $100 \mathrm{ml}$ ( $p$ value: $0.004,0.0035,0.001,0.001$ and 0.017 , compared to perforations of the stomach).

\section{Dependency of perforation location and amount of ascites}

All of the study patients exhibited a certain amount of ascites (100\% prevalence). There was significantly more ascites in the abdomen when the perforation was located in the upper GIT (median: $333 \mathrm{ml}$, percentiles $25-75 \%=70-750 \mathrm{ml}$ ) than when it was located in the lower GIT (median $100 \mathrm{ml}$, percentiles $25-75 \%=31-333 \mathrm{ml}, p=0.022$ ). Most ascites was found with perforations of the stomach, duodenum and ileum (median: 417, 333, $250 \mathrm{ml}$, entire statistics in Table 1 and Fig. 5, typical examples in Fig. 6). The presence of significantly less ascites indicated perforations of the large bowel (Fig. 7), e.g., a perforation in the ascending colon yielded an ascites volume of only $70 \mathrm{ml}(p=0.004$, compared to a perforation of the stomach).

\section{Relationship of free air and ascites dependent on the perforation location}

The delta of air and ascites volumes $\left(V_{\text {air }}-V_{\text {asc }}\right)$ and the sum of both volumes also demonstrated significant differences between the upper and lower GITs ( $p=0.005$ and $p=0.037$ ). However, the $p$ value of the ascites difference in upper and lower GIT perforations alone was lower $(p=0.0023)$. On the scatterplot diagrams the relation between free air and ascites is shown dependent on the perforation location (Fig. 8). In addition, the volume ratio of air and ascites between the upper and lower GIT perforations or the fact that there was more ascites than air (ascites scenario) did not lead to significant differences $(p=0.18, p=0.31)$.

\section{ROC analysis of ascites and air}

The AUC was $0.63 \pm 0.04$ using the amount of ascites $(\mathrm{ml})$ for differentiating the perforation location (upper vs lower GIT, Fig. 9). A cutoff level of $333 \mathrm{ml}$ ascites was the best criterion for location detection. When a perforation (free air) presented with $333 \mathrm{ml}$ ascites or less, it was more likely a lower GIT perforation (large bowel). The odds ratio for a perforation in the lower GIT when demonstrating less than $333 \mathrm{ml}$ ascites was 3.52 (95\% CI 2.7-4.0). This threshold led to a sensitivity and specificity for large bowel perforation of $80.7 \%$ and $45.6 \%$, respectively.

Using the volume of free air, a cutoff value of $70 \mathrm{ml}$ or less indicated a lower GIT perforation. However, the odds ratio for a perforation in the lower GIT with less than $70 \mathrm{ml}$ free air was only 1.76 (95\% CI 1.3-2.0); and the AUC was lower $(0.58 \pm 0.05)$ with a sensitivity and a specificity of $53.8 \%$ and $67.9 \%$, respectively.

\section{Sensitivity and confidence of radiologists for perforation location}

Radiologists scored a higher sensitivity for estimating the perforation location when the upper GIT (stomach, duodenum, jejunum and ileum) and the lower GIT (ascending, transverse, descending and sigmoid colon with rectum) were pooled together (sensitivity $=0.91 \pm 0.04$ ). When they had to guess the individual parts of the GIT, the 
Fig. 6 A 32-year-old Woman with a Perforation of the Stomach. Yielding typically large amounts of free air (asterisk) and ascites (arrows). Perforation captured on axial slice (A) and on sagittal reformat $(\mathbf{B})$
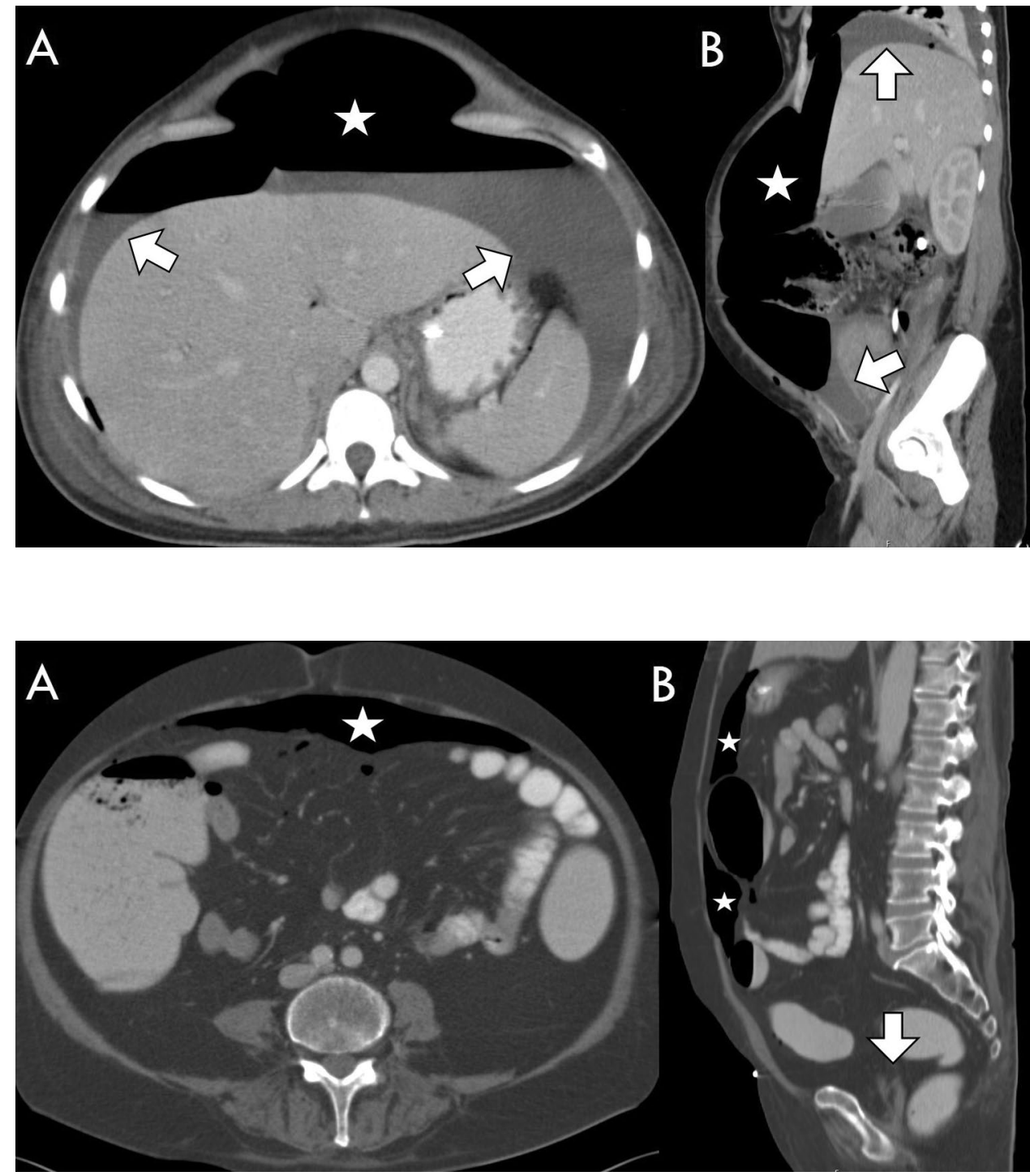

Fig. 7 A 58-year-old man with a perforation of the descending colon. Yielding a typically large amount of free air (asterisk) and only a small volume of ascites (arrow). Perforation captured on axial slice (A) and on sagittal reformat $(\mathbf{B})$
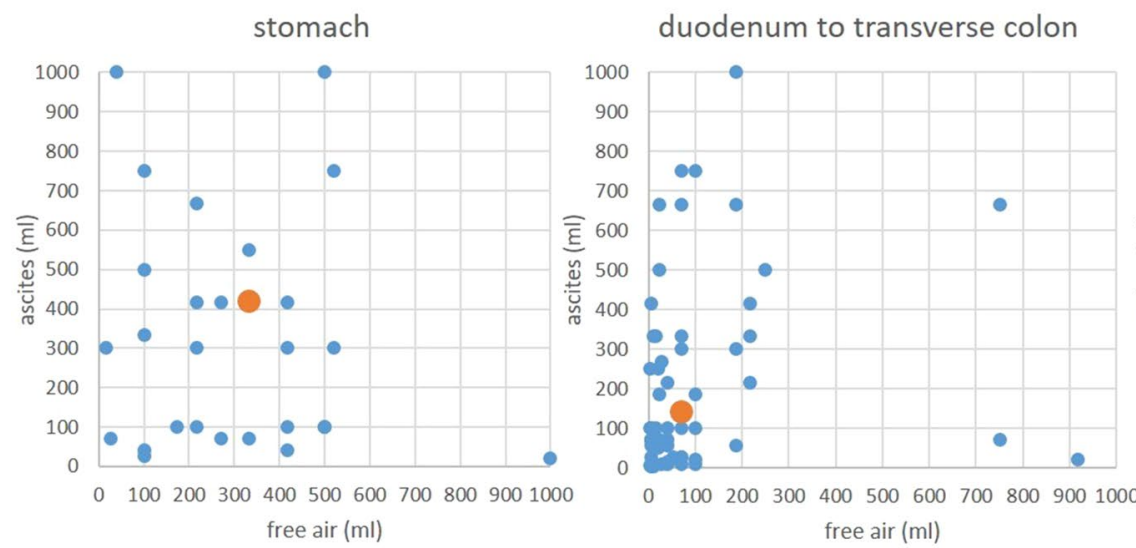

descending and sigmoid colon

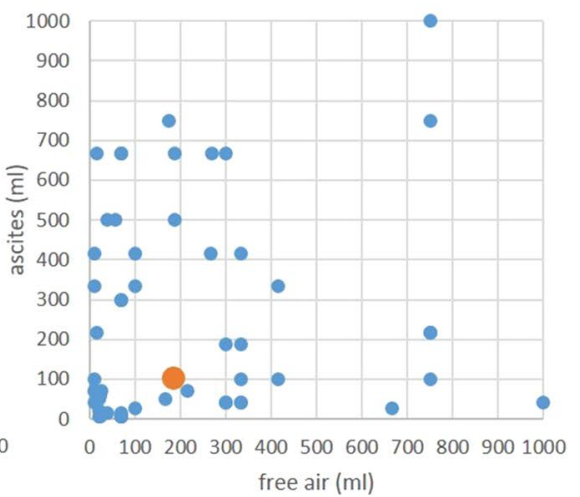

Fig. 8 Scatterplot of the volume of free air versus the volume of ascites per location: perforations of the stomach demonstrated the largest volume of free air and ascites (median is shown as orange point), while perforations of the middle GIT lead to smaller volumes (median in orange). Leakage of the descending and sigmoid colon demonstrated more free air than ascites (median in orange). Only volumes smaller than $1000 \mathrm{ml}$ are shown 


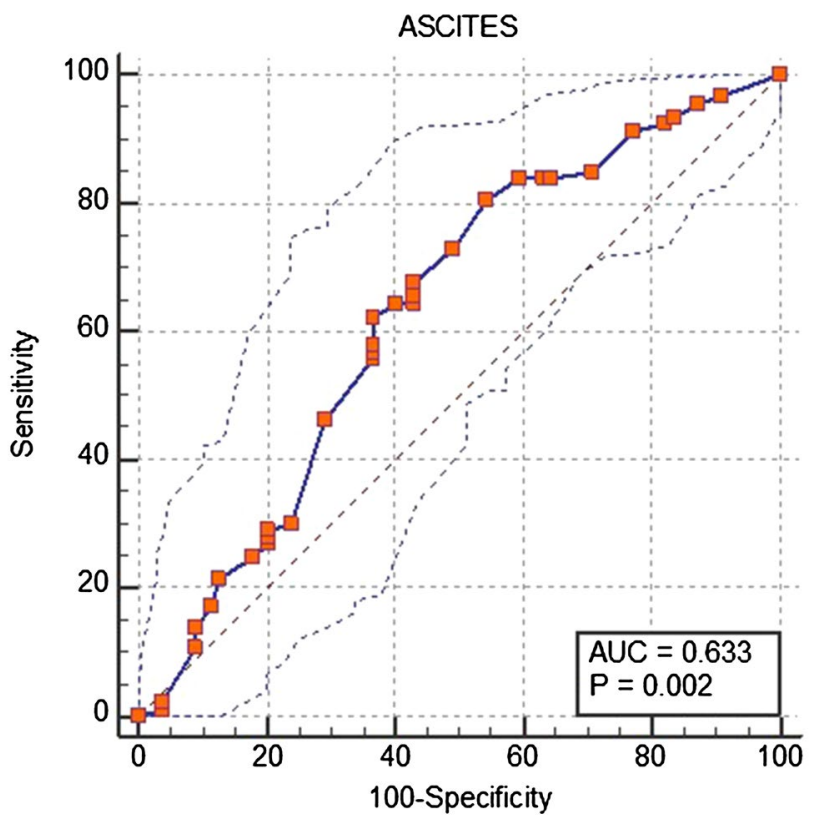

Fig. 9 ROC, AUC, sensitivity and specificity of ascites for differentiating upper from lower GIT perforation

Table 2 Sensitivity of the radiologists for perforation location

\begin{tabular}{llll}
\hline & SENS 1-8 & SENS (up/lo GIT) & Confidence GIT 1-8 \\
\hline R1 & 0.75 & 0.93 & 3.8 \\
R2 & 0.59 & 0.89 & 3.8 \\
R3 & 0.61 & 0.86 & 3.4 \\
R4 & 0.79 & 0.96 & 3.6 \\
All & $0.68( \pm 0.09)$ & $0.91( \pm 0.04)$ & $3.6( \pm 0.2)$ \\
\hline
\end{tabular}

$R$ reader, SENS sensitivity, sd standard deviation, up/lo GIT upper/ lower gastrointestinal tract sensitivity dropped to $0.68( \pm 0.09)$ at a relatively high confidence level of $3.6( \pm 0.2)$, meaning that the radiologists were "reasonably sure" of their location prediction (Table 2).

\section{Volumetry (man versus machine) and interobserver agreement among radiologists}

The correlation coefficients (CCs) between semiautomated volume measurements and volume estimates of raters 1 , 2, 3, and 4 were 0.96 (CI 0.93 to 0.98 ), 0.75 (CI 0.53 to 0.87 ), 0.82 (CI 0.67 to 0.91 ) and 0.91 (CI 0.81 to 0.95 ) for ascites, respectively, with a pooled coefficient for all raters of 0.80 (CI 0.72 to 0.85 ). For air volume measurement, the $\mathrm{CC}$ between the machine and the radiologists was 0.87 (CI 0.76 to 0.94 ), 0.75 (CI 0.55 to 0.86 ), 0.78 (CI 0.58 to 0.89 ), and 0.85 (CI 0.71 to 0.93 ), with a pooled CC of 0.80 (CI 0.72 to 0.85 ). On average, radiologists measured $57 \pm 474 \mathrm{ml}$ more air and $64 \pm 379 \mathrm{ml}$ more ascites for each case compared to the ground truth (semiautomated volumetry, Fig. 10).

The interobserver agreement between rater 1 and rater 2 for the volume of free air was $0.69 \pm 0.05$, and their interobserver agreement for the volume of ascites was $0.55 \pm 0.05$. The agreement on the perforation location was $0.81 \pm 0.04$. For raters 3 and 4 , the agreements for air volume, ascites volume and perforation location were $0.64 \pm 0.05,0.68 \pm 0.04$, and $0.81 \pm 0.06$, respectively. Overall, substantial agreement between air and ascites estimates by the naked eye was reached $(0.64 \pm 0.05)$, and for the perforation location, almost perfect agreement could be scored $(0.81 \pm 0.05)$. There was no difference in correct classification between the more experienced radiologists $(1 / 2)$ and moderately experienced radiologists $(3 / 4)(p=0.4)$.

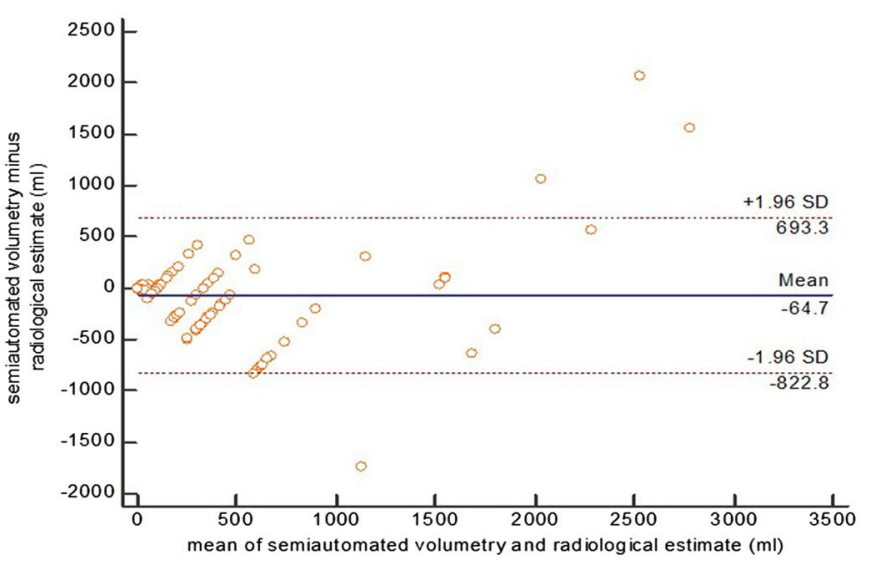

Fig. 10 Bland-Altman plots: volume differences between machine and radiologist for air (left side) and ascites (right side) 


\section{Discussion}

Our results demonstrate that both ascites and free air volumes are larger in upper GIT perforations. Radiologists could very accurately name the location of the perforation using their experienced skills for detection of the smallest air bubbles around the perforation or the detection of wall defects. Experienced radiologists are advantageous, but for residents and fellows on night shifts in an emergency room, determining the amount of ascites may help to find the perforation location, which may be very useful for visceral surgeons.

Determining the volume of ascites might boost the confidence level of radiologists in finding the perforation location. With the application of a simple rule, perforation locations can be classified as upper or lower GIT. The rule says that one must estimate whether the ascites volume is more or less than $333 \mathrm{ml}$, which is the exact volume of a soda can. This approach helps radiologists image and compare liquid volumes. In comparison to the semiautomated volume measurement, the radiologists reached a high agreement with the machine that was comparable to the agreement of one radiologist with another. However, the radiologists estimated the volumes to be slightly higher (by $55-65 \mathrm{ml}$ ). Since the largest amount of free air is seen in perforations at the beginning and at the end of the GIT (stomach, descending and sigmoid colon) and ascites is found more in the presence of upper GIT perforations, the ratio of the sum or the delta of air and ascites is obviously not as helpful as the amount of ascites alone for localizing the perforation.

The two most frequent sites of perforation were sigmoid colon $(32.6 \%)$ and stomach $(20.3 \%)$, combined they represented more than $50 \%$ of all our study cases. With this information alone radiologists should know where to start looking for a leak in the GIT. The fact that perforations in the lower GIT demonstrated less amount of ascites, helps separating the two locations. It needs to be indicated that there was an overlap between air and fluid volume between the stomach and the sigmoid colon as shown in Figs. 4 and 5. Therefore, sensitivity and AUC of the proposed volume cutoffs were never $100 \%$ for classifying the perforations into upper and lower GIT perforations.

In the future, the following two-step algorithm needs to be investigated (Fig. 11):

1. The amount of free air determines whether the perforation is located in the middle of the GIT or not.

2. The volume of ascites then determines if the perforation location is in the upper or lower GIT.

When the classification of the radiologists was pooled into the 4 segments suggested by the 2 step algorithm

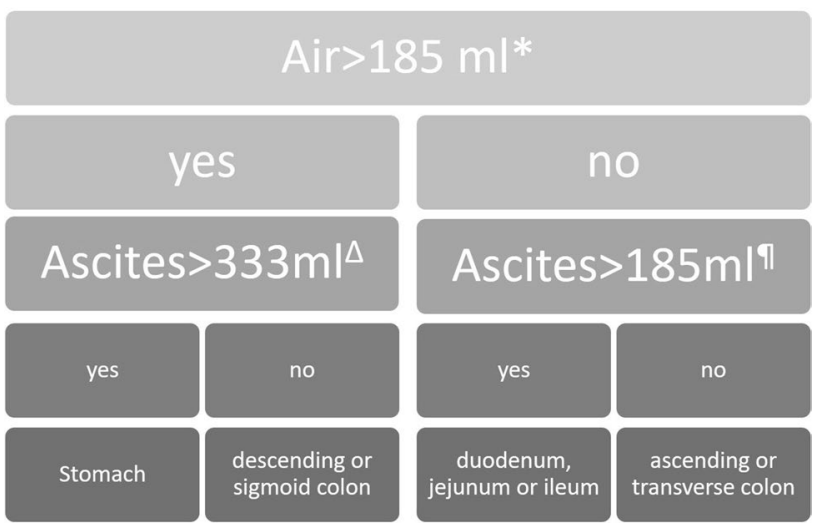

Fig. 11 Perforation location flow chart. *A cutoff value of air volume $>185 \mathrm{ml}$ led to a sensitivity, specificity and AUC of 56.1, 73.0 and $0.64 \pm 0.04$ for classification into stomach, descending or sigmoid colon perforations. ${ }^{\Delta}$ The threshold of ascites $>333 \mathrm{ml}$ then demonstrated a sensitivity, specificity and AUC of 51.4, 79.4 and $0.69 \pm 0.05$ for distinguishing stomach versus descending or sigmoid colon perforations, respectively. " Ascites volumes over $185 \mathrm{ml}$ could classify the middle GIT into the duodenum, jejunum or ileum with a sensitivity, specificity and AUC of 58.7, 71.4 and $0.59 \pm 0.07$, respectively

(Fig. 11), the four readers together misclassified the perforation location in 69 cases. In these cases the readers demonstrated low confidence, and the proposed algorithm detected 28 correct locations of perforation $(=40.6 \%, p$ value was 0.0458 compared to chance $(25 \%)$ ). Previous reports have emphasized CT manifestations of bowel perforation secondary to various causes. However, no previous reports have tried to quantify the most useful findings as free air and fluid. For example, Ongolo-Zogo et al. [27] reported on a series of 10 perforated gastroduodenal peptic ulcers in which two important CT findings were indicative of the site of perforation: discontinuity in the bowel wall in six patients and tiny extraluminal air bubbles in close proximity to the bowel wall in two patients. Miki et al. [28] also reported direct visualization of a ruptured colonic wall in four of six patients with colonic perforation. In the study of Hainaux et al. [21], the authors concentrated on free air bubbles in close proximity to the bowel wall and segmental bowel wall thickening as strong predictors of perforation at the site.

Our approach differs from that in the study of Seishima et al. [29], in which the author retrospectively concentrated on the CT attenuation values of ascites, demonstrating a higher density of ascites in patients with colorectal perforation than in those with perforations at other sites.

The study of Shanmuganathan et al. [30] shows that helical CT with administration of rectal, oral, and IV contrast material is highly accurate for evaluating patients with penetrating injuries to the thoracoabdominal region. Nevertheless, only $15 \%$ of patients with bowel injury showed oral or rectal contrast material extravasation. In our study, we 
focused mainly on patients with nontraumatic bowel perforations. In such patients, it is often difficult to obtain opacification of the ascites, future studies will have to investigate the density of ascites and perforation location in traumatic and nontraumatic patients. We halfway confirmed the hypothesis that upper GIT perforations yield more free air, but we had to reject the hypothesis that lower GIT perforations would result in more ascites. Our results showed the exact opposite. Our aim was to devise a simple rule that can always be applied without complicated time-consuming measurements at every emergency CT imaging unit, based on the fact that retrospective identification of the site of perforation helps the emergency department physician plan the appropriate treatment in a potentially unstable patient and assists the surgeon in planning the correct surgical approach.

\section{Limitations}

We did not consider the delay between the time of onset of symptoms and the time of CT examination. Potential peritonitis caused by either upper or lower GIT perforations may lead to more ascites over time, which could confound our results. Our results represent the measurements in a consecutive population of perforation patients in a tertiary care hospital center. Factors other than location are not included in this study such as size of the perforation, density of ascites or whether the location was intraperitoneal or extraperitoneal. We wanted to focus on the many cases where the location could not primarily be identified by a large interruption of the bowel wall or by small gas bubbles around an extraperitoneal or covered perforation.

\section{Conclusions}

Our results demonstrate that the amount of free air is larger in upper GI and distal lower GI perforations than in other sites of the gut, and upper GI perforations have a greater volume of ascites. Using a two-step process based on the volumes of free air and free fluid can accurately localize the site of perforation to the upper or lower GI tract. When used in conjunction with other CT findings, such as location of small extraluminal gas bubbles, these findings can increase the confidence of the radiologist in identification of the site of bowel perforation. This algorithm may be especially helpful for residents or junior attendings in diagnosing the perforation site.

Such information is of vital assistance to the visceral surgeon.

Acknowledgements We would like to thank the highly motivated team of medical technicians in our image lab, Michelle Schweizer and Géraldine Gemmet, who contributed many days for CT volumetry.
Funding Open Access funding provided by Universität Bern. There was no funding of this study.

Data availability Data are available upon special request.

\section{Declarations}

Conflict of interest The authors declare that they have no conflict of interest.

Ethical approval The Cantonal Ethics Committee approved this retrospective study (Ethics Approval Nr. 2020-01279).

Informed consent Due to the retrospective data collection and anonymization the consent could be waived according to the ethics approval.

Open Access This article is licensed under a Creative Commons Attribution 4.0 International License, which permits use, sharing, adaptation, distribution and reproduction in any medium or format, as long as you give appropriate credit to the original author(s) and the source, provide a link to the Creative Commons licence, and indicate if changes were made. The images or other third party material in this article are included in the article's Creative Commons licence, unless indicated otherwise in a credit line to the material. If material is not included in the article's Creative Commons licence and your intended use is not permitted by statutory regulation or exceeds the permitted use, you will need to obtain permission directly from the copyright holder. To view a copy of this licence, visit http://creativecommons.org/licenses/by/4.0/.

\section{References}

1. Ghahremani GG (1993) Radiologic evaluation of suspected gastrointestinal perforations. Radiol Clin North Am 31:1219-1234

2. Maniatis V, Chryssikopoulos H, Roussakis A, Kalamara C, Kavadias S, Papadopoulos A, Andreou J, Stringaris K (2000) Perforation of the alimentary tract: evaluation with computed tomography. Abdom Imaging 25:373-379. https://doi.org/10. $1007 / \mathrm{s} 002610000022$

3. Jawad H, Raptis C, Mintz A, Schuerer D, Mellnick V (2018) Single-Contrast CT for Detecting Bowel Injuries in Penetrating Abdominopelvic Trauma. AJR Am J Roentgenol 210(4):761765. doi: https://doi.org/10.2214/AJR.17.18496.

4. Saturnino PP, Pinto A, Liguori C, Ponticiello G, Romano L (2016) Role of Multidetector Computed Tomography in the Diagnosis of Colorectal Perforations. Semin Ultrasound CT MR 37(1):49-53. doi: https://doi.org/10.1053/j.sult.2015.10.007

5. Simonetti I, Puglia M, Tarotto L, Palumbo F, Esposito F, Sciuto A, Palumbo L, Ragozzino A (2019) When traditions become dangerous: Intestinal perforation from unusual foreign bodyCase report and short literature review. Eur J Radiol Open 6:152-155. doi: https://doi.org/10.1016/j.ejro.2019.04.002

6. Wang R, He J, Chen Z, Wen K (2021) Migration of fish bones into abdominal para-aortic tissue from the duodenum after leading to duodenal perforation: a case report. BMC Gastroenterol 21(1):82. doi: https://doi.org/10.1186/s12876-021-01662-3

7. Bielecki K, Kamiński P, Klukowski M (2002) Large bowel perforation: morbidity and mortality. Tech Coloproctol 6:177-182. https://doi.org/10.1007/s101510200039 
8. Kriwanek S, Armbruster C, Beckerhinn P, Dittrich K (1994) Prognostic factors for survival in colonic perforation. Int J Colorectal Dis 9:158-162. https://doi.org/10.1007/bf00290194

9. Pisanu A, Cois A, Uccheddu A (2004) Surgical treatment of perforated diverticular disease: evaluation of factors predicting prognosis in the elderly. Int Surg 89:35-38

10. Ohmann C, Wittmann DH, Wacha H (1993) Prospective evaluation of prognostic scoring systems in peritonitis. Peritonitis study group. Eur J Surg 159:267-274

11. Miller RE, Nelson SW (1971) The roentgenologic demonstration of tiny amounts of free intraperitoneal gas: experimental and clinical studies. Am J Roentgenol Radium Ther Nucl Med 112:574-585. https://doi.org/10.2214/ajr.112.3.574

12. Cho KC, Baker SR (1994) Extraluminal air. Diagnosis and significance. Radiol Clin North Am 32:829-844

13. Rice RP, Thompson WM, Gedgaudas RK (1982) The diagnosis and significance of extraluminal gas in the abdomen. Radiol Clin North Am 20:819-837

14. Chen SC, Wang HP, Chen WJ, Lin FY, Hsu CY, Chang KJ, Chen WJ (2002) Selective use of ultrasonography for the detection of pneumoperitoneum. Acad Emerg Med 9:643-645. https://doi.org/ 10.1111/j.1553-2712.2002.tb02307.x

15. Rajaguru K, Sheong SC (2020) Case report on a rare cause of silent duodenal perforation. Int J Surg Case Rep 76:320-323. doi: https://doi.org/10.1016/j.ijscr.2020.09.184

16. Yeung KW, Chang MS, Hsiao CP, Huang JF (2004) CT evaluation of gastrointestinal tract perforation. Clin Imaging 28:329-333. https://doi.org/10.1016/s0899-7071(03)00204-3

17. Stapakis JC, Thickman D (1992) Diagnosis of pneumoperitoneum: abdominal CT vs upright chest film. J Comput Assist Tomogr 16:713-716

18. Earls JP, Dachman AH, Colon E, Garrett MG, Molloy M (1993) Prevalence and duration of postoperative pneumoperitoneum: sensitivity of CT vs left lateral decubitus radiography. AJR 161:781785. doi: https://doi.org/10.2214/ajr.161.4.8372757

19. Catalano O (1996) Computed tomography in the study of gastrointestinal perforation. Radiolo Med (Torino) 91:247-252

20. Kia M, MacDonald TL, Iddings D (2014) Nonsurgical pneumoperitoneum. Appl Radiol 14(9):44-46

21. Hainaux B, Agneessens E, Bertinotti R, De Maertelaer V, Rubesova E, Capelluto E, Moschopoulos C (2006) Accuracy of MDCT in predicting site of gastrointestinal tract perforation. AJR Am J Roentgenol 187:1179-1183. https://doi.org/10.2214/ajr.05.1179
22. Múnera F, Morales C, Soto JA, et al. (2004) Gunshot wounds of abdomen: evaluation of stable patients with triple-contrast helical CT. Radiology 231:399-405. doi/https://doi.org/10.2214/AJR.17. 18496

23. Himmelman RG, Martin M, Gilkey S, Barrett JA (1991) Triplecontrast CT scans in penetrating back and flank trauma. J Trauma 31:852-855. doi/https://doi.org/10.2214/AJR.17.18496

24. Stoker J, van Randen A, Laméris W, Boermeester MA (2009) Imaging patients with acute abdominal pain. Radiology 253(1):31-46. doi: https://doi.org/10.1148/radiol.2531090302

25. Huynh LN, Coughlin BF, Wolfe J, Blank F, Lee SY, Smithline HA (2004) Patient encounter time intervals in the evaluation of emergency department patients requiring abdominopelvic CT: oral contrast versus no contrast. Emerg Radiol 10:310 -313. doi: https://doi.org/10.1007/s10140-004-0348-1

26. Laméris W, van Randen A, van Es HW, et al. (2009) Imaging strategies for detection of urgent conditions in patients with acute abdominal pain: diagnostic accuracy study. BMJ 338:b2431. doi: https://doi.org/10.1136/bmj.b2431.

27. Ongolo-Zogo P, Borson O, Garcia P, Gruner L, Valette PJ (1999) Acute gastroduodenal peptic ulcer perforation: contrast-enhanced and thin-section spiral CT findings in 10 patients. Abdom Imaging 24:329-332. https://doi.org/10.1007/s002619900509

28. Miki T, Ogata S, Uto M, Nakazono T, Urata M, Ishibe R, Shinyama S, Nakajo M (2004) Multidetector-row CT findings of colonic perforation: direct visualization of ruptured colonic wall. Abdom Imaging 29:658-662. https://doi.org/10.1007/s00261-003-0159-y

29. Seishima R, Okabayashi K, Hasegawa H, Tsuruta M, Hoshino H, Yamada T, Kitagawa Y (2015) Computed tomography attenuation values of ascites are helpful to predict perforation site. World J Gastroenterol 21:1573-1579. https://doi.org/10.3748/wjg.v21.i5. 1573

30. Shanmuganathan K, Mirvis SE, Chiu WC, Killeen KL, Scalea TM (2001) Triple-contrast helical CT in penetrating torso trauma: a prospective study to determine peritoneal violation and the need for laparotomy. AJR Am J Roentgenol 177:1247-1256. https://doi. org/10.2214/ajr.177.6.1771247

Publisher's Note Springer Nature remains neutral with regard to jurisdictional claims in published maps and institutional affiliations. 


\section{Authors and Affiliations}

\section{Dionysios Drakopoulos ${ }^{1}$. Jacqueline Arcon ${ }^{1} \cdot$ Peter Freitag ${ }^{1} \cdot$ Mostafa El-Ashmawy $^{1}$ - Steven Lourens ${ }^{1}$. Guido Beldi ${ }^{2} \cdot$ Verena Carola Obmann ${ }^{3}$ (1) $\cdot$ Lukas Ebner $^{3}$ (1) $\cdot$ Adrian Thomas Huber $^{3}$ (1) $\cdot$ Andreas Christe $^{1,3}$ (1)}

\author{
Andreas Christe \\ andreas.christe@insel.ch \\ Dionysios Drakopoulos \\ dionysios.drakopoulos@insel.ch \\ Jacqueline Arcon \\ jjarcon@hotmail.com \\ Peter Freitag \\ peter.freitag@insel.ch \\ Mostafa El-Ashmawy \\ mostafa.elashmawy@insel.ch \\ Steven Lourens \\ steven.lourens@insel.ch \\ Guido Beldi \\ guido.beldi@insel.ch
}

\author{
Verena Carola Obmann \\ verena.obmann@insel.ch \\ Lukas Ebner \\ lukas.ebner@insel.ch
}

Adrian Thomas Huber adrian.huber@insel.ch

1 Department of Diagnostic, Interventional and Pediatric Radiology, INSELGROUP, Radiology Division SLS, University of Bern, Tiefenaustrasse 112, 3004 Bern, Switzerland

2 Department of Visceral and Transplantation Surgery, Inselspital, Bern University Hospital, University of Bern, Freiburgstrasse 10, 3010 Bern, Switzerland

3 Department of Diagnostic, Interventional and Pediatric Radiology, Inselspital, Bern University Hospital, University of Bern, Freiburgstrasse 10, 3010 Bern, Switzerland 